\title{
Temperature dependent phase stability of nanolaminated ternaries from first-principles calculations
}

\author{
Andreas Thore, Martin Dahlqvist, Björn Alling and Johanna Rosén
}

\section{Linköping University Post Print}

\section{Tweet}

N.B.: When citing this work, cite the original article.

Original Publication:

Andreas Thore, Martin Dahlqvist, Björn Alling and Johanna Rosén, Temperature dependent phase stability of nanolaminated ternaries from first-principles calculations, 2014, Computational materials science, (91), 251-257.

http://dx.doi.org/10.1016/j.commatsci.2014.04.055

Copyright: Elsevier http://www.elsevier.com/

Postprint available at: Linköping University Electronic Press http://urn.kb.se/resolve?urn=urn:nbn:se:liu:diva-109360 


\title{
Temperature dependent phase stability of nanolaminated ternaries from first-principles calculations
}

\author{
A. Thore ${ }^{a, *}$, M. Dahlqvist ${ }^{a}$, B. Alling ${ }^{a}$, and J. Rosén ${ }^{a}$ \\ a Department of Physics, Chemistry, and Biology, Thin film physics division, Linköping University, \\ SE-581 83 Linköping, Sweden
}

*Corresponding author. Tel.: +4670 3212109 .

E-mail address: andth@ifm.liu.se

E-mail addresses to co-authors:

M. Dahlqvist: madah@ifm.liu.se

B. Alling: bjoal@ifm.liu.se

J. Rosén: johro@ifm.liu.se

\begin{abstract}
Methods based on first-principles calculations have proven effective for predicting the thermodynamic stability of materials that have not previously been considered. However, the vast majority of these predictions are based on $0 \mathrm{~K}$ calculations, which means that little is known about the effects of temperature on their accuracy. This causes considerable uncertainty with respect to stability predictions of new hypothetical phases.

In this work we combine first-principles calculations with an optimization procedure to calculate the phase stability as a function of temperature for $\mathrm{Ti}_{2} \mathrm{AlC}_{2} \mathrm{Ti}_{3} \mathrm{AlC}_{2}$ and $\mathrm{Ti}_{4} \mathrm{AlC}_{3} \mathrm{MAX}$ phases with respect to their most competing phases in the Ti-Al-C phase diagram, in a temperature interval from 0 to 2000 $K$. To model nonzero temperatures, we include effects from the electronic and vibrational free energies to the Gibbs free energy for all relevant competing phases. We show that, due to a mutual cancellation of the temperature dependent energy terms, the results of neither the harmonic nor the quasiharmonic calculations differ significantly from the calculated $0 \mathrm{~K}$ formation energies. We thus provide a plausible explanation for the success of previous $0 \mathrm{~K}$ predictions, an explanation which also serves as evidence for the hypothesis that the phase stability in many materials systems is primarily governed by the $0 \mathrm{~K}$ energy terms.
\end{abstract}

\section{Keywords}

First-principles; phase stability; ternary carbides; harmonic approximation; quasiharmonic approximation; density functional theory

\section{Introduction}

Since its discovery in the 1960s by Nowotny [1], the class of so-called MAX phases has been subjected to extensive theoretical and experimental research. The general formula of these nanolaminated, hexagonally structured materials is $M_{n+1} A X_{n}$, where $M$ is a transition metal, $A$ is an $A$ group element, and $X$ is most commonly carbon and/or nitrogen. Due to the metallic $M-A$ bonds and ionic-covalent like $M-X$ bonds, $M A X$ phases possess both properties typical of metals such as good machinability and high electrical as well as high thermal conductivity, and ceramic properties such as high hardness and resistance against corrosion and the wearing effects of high temperatures $[2,3]$. Because of this, MAX phases show potential for, e.g., electrical contacts, tool coatings, and as structural material in demanding environmental conditions. 
A useful tool in the search for new and not yet experimentally verified materials - and in this particular case, MAX phases - is a computational method described in detail in two papers by Dalhqvist et al. [4, 5]. It combines first-principles calculations with a linear optimization procedure to find the set of most competing phases at any compositional point of interest in a multinary space. Equivalent approaches have also been used to search for new thermodynamically stable phases in other multicomponent systems such as Heusler alloys and oxynitrides [6, 7]. The method has been used to retrodict and to successfully predict the existence of several MAX phases, and also to explain failed synthesis attempts of hypothetical MAX phases. The number of retrodicted MAX phases include, e.g, $\mathrm{Cr}_{2} \mathrm{AlC}, \mathrm{Ti}_{2} \mathrm{AlC}, \mathrm{Ti}_{3} \mathrm{AlC}_{2}, \mathrm{Ti}_{2} \mathrm{AIN}, \mathrm{Ti}_{4} \mathrm{AlN}_{3}, \mathrm{Ti}_{3} \mathrm{SiC}_{2}, \mathrm{~V}_{2} \mathrm{AlC}, \mathrm{V}_{3} \mathrm{AlC}_{2}$ and $\mathrm{V}_{4} \mathrm{AlC}_{3-\delta}[5,8]$. Examples of predicted and later experimentally verified phases are $\mathrm{Nb}_{2} \mathrm{GeC},\left(\mathrm{Cr}_{1-\mathrm{x}} \mathrm{Mn}_{\mathrm{x}}\right)_{2} \mathrm{AlC},\left(\mathrm{Cr}_{1-\mathrm{x}} \mathrm{Mn}_{\mathrm{x}}\right)_{2} \mathrm{GeC}$, and $\mathrm{Mn}_{2} \mathrm{GaC}$ [9-12]. However, while this serves as evidence that the method is reliable, there are potential pitfalls that need to be considered.

As in the above examples, most first-principles based phase stability calculations are performed at 0 $\mathrm{K}$. This means that neither the electronic nor the vibrational (i.e., phonon) entropic contributions to the total energy are taken into account. However, configurational entropy is sometimes considered in the case of alloys $[11,13]$. Considering temperature induced excitations could be necessary if the difference in energy between the investigated phase and its most competing phases is otherwise small enough to be comparable in size to the errors introduced by the finite accuracy of the calculations. As is noted by Dahlqvist et al. [5], for the hypothetical case of $\mathrm{Ti}_{4} \mathrm{AlC}_{3}$, including temperature dependent contributions in the calculations might play an essential role in determining whether this phase is stable or not. Another case could be if a particular phase, which is thermodynamically or even dynamically unstable at low temperatures, is stabilized and becomes important as a competing phase at high temperatures.

Calculations of various physical properties of crystals at nonzero temperatures are not nearly as commonly reported in the literature as $0 \mathrm{~K}$ calculations due to the higher theoretical complexity and much higher computational demands, stemming mainly from the vibrational contribution to the formation energy. Nevertheless, due to technological advances such calculations are rapidly becoming practically feasible, and in recent years several MAX phase related theoretical studies dealing with temperature dependence have been published. While most of these studies have been dedicated to the characterization of thermodynamic properties such as heat capacity, thermal expansion, and bulk modulus [14-18], some of them have indeed focused on phase stability. However, their scopes have been limited to investigations of the relative stability between $\alpha$ and $\beta$ MAX phase polymorphs [19-21].

In this work, we extend the $0 \mathrm{~K}$ method described in Ref. [5] into a temperature dependent phase stability criterion based on the Gibbs free energy within the so-called harmonic and quasiharmonic approximations (HA and QHA). We investigate the phase stability of the $\mathrm{Ti}_{n+1} \mathrm{AlC}_{n}$ (for $n=1-3$ ) MAX phases with respect to competing phases in the Ti-Al-C phase diagram, in a temperature interval from 0 to $2000 \mathrm{~K}$. The HA is used to model all phases, and for a carefully selected subset we also use the QHA for comparison. We show that despite the inclusion of both the electronic and the vibrational contributions to the Gibbs free energies, neither within the HA nor the QHA are the calculated phase stabilities significantly affected as compared to the $0 \mathrm{~K}$ case. This result thus provides further evidence that it is mainly the $0 \mathrm{~K}$ energy terms that govern phase stability, or, stated 
differently, that $0 \mathrm{~K}$ calculations can indeed be used for confident predictions of the existence of new MAX phases.

\section{Methodology}

\section{a. Thermodynamic phase stability}

If the electron-phonon interactions are assumed to be negligible, the Gibbs free energy can be written as

$$
G(p, T)=E_{0}(V)+F_{e l}(T, V)+F_{v i b}(T, V)+F_{c}(T)+p V,
$$

where $E_{0}(V)$ is the zero-temperature energy of the crystal, i.e., the energy that the phase would have at $0 \mathrm{~K}$ at volume $\mathrm{V}$, and $F_{e l}, F_{v i b}$, and $F_{c}$ are the free electronic, vibrational and configurational energies, respectively. The last term in Eq. (1) has been neglected in the present work, as the ambient pressure was set to $0 \mathrm{GPa}$.

The free electronic energy $F_{e l}$ is given by the difference between the electronic excitation energy, $E_{e l}$, and the entropic contribution, $T S_{e l}$ :

$$
F_{e l}(V, T)=E_{e l}(V, T)-T S_{e l}(V, T) .
$$

$E_{e l}$ can be found by subtracting the total electronic energy in the excited state with the electronic ground state energy,

$$
E_{e l}(V, T)=\int_{0}^{\infty} n(\varepsilon, V) \varepsilon f(\varepsilon) d \varepsilon-\int_{0}^{\varepsilon_{f}} n(\varepsilon, V) \varepsilon d \varepsilon
$$

where $n(\varepsilon, V)$ is the electronic density of states, $f(\varepsilon)$ the Fermi-Dirac distribution, and $\varepsilon_{F}$ the Fermi energy.

The electronic entropy $S_{e l}$ can be expressed as

$$
S_{e l}(V, T)=-k_{B} \int n(\varepsilon, V)[(f(\varepsilon) \ln f(\varepsilon)+(1-f(\varepsilon)) \ln (1-f(\varepsilon))] d \varepsilon .
$$

The vibrational free energy in Eq. (1) is given by

$$
F_{v i b}(V, T)=\frac{1}{2} \sum_{q, v} \hbar \omega_{q, v}+k_{B} T \sum_{q, v} \ln \left[1-\exp \left(-\hbar \omega_{q, v} / k_{B} T\right)\right]
$$

where $\omega$ is the phonon frequency, $\boldsymbol{q}$ is the wave vector, and $v$ is the band index [14].

Finally, the free configurational energy $F_{c}$ can be written as

$$
F_{c}(T)=E_{c}(T)-T S_{c}(T),
$$

for which the mean field approximation (MF) often gives a good estimate, with $E_{c}=E_{\text {random }}-E_{o}$ and 


$$
S_{c}^{M F}=-k_{B} \sum_{i}^{n} y_{i} \ln y_{i}
$$

where $y_{i}$ is the mole fraction of species $i$. Here $E_{\text {random }}$ is the energy of an ideally disordered random alloy that can be modeled with, e.g., the special quasirandom structure (SQS) technique while $S_{c}^{M F}$ is the corresponding configurational entropy of an ideal solid solution. However, with one single exception the phases included in this work are compositionally ordered, which means that $F_{c}$ is zero.

In the harmonic approximation, the volume dependence of $G$ (i.e., thermal expansion) is neglected. Using the HA speeds up the calculations significantly, but the results are less accurate as compared to the quasiharmonic approximation, under which the volume dependence of $G$ is included.

Once the Gibbs free energy for all relevant phases have been calculated, a linear optimization procedure is employed in order to determine whether the phase of interest is stable or not with respect to any combination of other competing phases. This procedure works by identifying the set of competing phases for which the sum of the respective Gibbs free energies is minimized. Thus, if the stability of a MAX phase is investigated we get

$$
\min G_{c p}\left(b^{M}, b^{A}, b^{X}\right)=\sum_{i}^{n} x_{i} G_{i}
$$

where $b^{M, A, X}$ is the amount of species $M, A$, and $X$ in the MAX phase, $x_{i}$ is the amount of compound $i$, and $G_{i}$ its free energy. The weighting factors $x_{i}$ must be chosen so that the total amount of each atomic species in the set of competing phases is the same as in the MAX phase. The constraint put on $x_{i}$ is such that

$$
x_{i} \geq 0 ; \quad \sum_{i}^{n} x_{i} b_{i}^{M}=b^{M}, \quad \sum_{i}^{n} x_{i} b_{i}^{A}=b^{A}, \quad \sum_{i}^{n} x_{i} b_{i}^{X}=b^{X} .
$$

The condition for thermodynamical stability is that

$$
\Delta G_{c p}=G_{M A X}-G_{c p}<0,
$$

i.e., the difference between the Gibbs free energy of the investigated phase and the Gibbs free energy for the identified set of most competing phases is less than zero. Here we note that some authors use the convention that the investigated phase itself is included in the set of competing phases, giving $\Delta G_{c p}=0$ for all stable phases and a positive "instability energy" for all other cases. However, such a procedure hides useful information of how stable, in terms of the magnitude of negative values of $\Delta G_{c p}$, the investigated phase is with respect to other phases. As all computational approaches involve approximations, a value of $\Delta G_{c p}$ that is just below zero might not guarantee successful experimental synthesis while a case where $\Delta G_{c p}$ is well below zero with several tenths of meV/atom is more promising. The definition used in e.g. Ref. [7] would not distinguish between these two cases.

\section{b. Calculation details}

All structural relaxations were carried out using the Vienna ab initio simulation package (VASP) [2225], which is an implementation of density functional theory (DFT) [26]. To solve the Kohn-Sham 
equations [27], the projector augmented wave (PAW) method was used [28], together with the Perdew-Burke-Ernzerhof generalized gradient approximation (PBE-GGA) for the exchange-correlation energy functional $[29,30]$. The plane wave cutoff energy was set to $400 \mathrm{eV}$, and all phases were converged to an accuracy of $0.1 \mathrm{meV} / \mathrm{atom}$. In order to sample k-space within the Brillouin zone, Monkhorst-Pack k-point grids were used for all phases.

The force constant matrices, which were used to determine the respective vibrational contributions, were calculated using density functional perturbation theory (DFPT) as implemented in VASP on appropriately large supercells [31]. With the help of the PHONOPY software these matrices were then used to sample a set of q-points, which finally yielded $F_{v i b}[32]$.

The supercells were constructed from the relaxed conventional unit cells of each phase, and they were then converged to an accuracy of within $0.5 \mathrm{meV} /$ atom both with respect to the number of $\mathrm{k}$ points and the forces due to the atomic displacements. Figure 1 shows the vibrational energy per atom (normalized with respect to $F_{\text {vib }}$ of the $4 \times 4 \times 1$ supercell) for $2 \times 2 \times 1$, a $3 \times 3 \times 1$, and a $4 \times 4 \times 1 \mathrm{Ti}_{2} \mathrm{AIC}$ supercells as a function of the length of the shortest side of the cell (denoted as $L_{\min }$ ) at 0,300 and $1000 \mathrm{~K}$. For $\mathrm{Ti}_{2} \mathrm{AlC}$, the force due to the displacement of an atom is still felt rather strongly by atoms

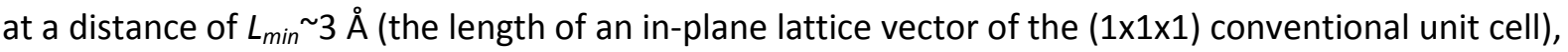
especially at 300 and $1000 \mathrm{~K}$.

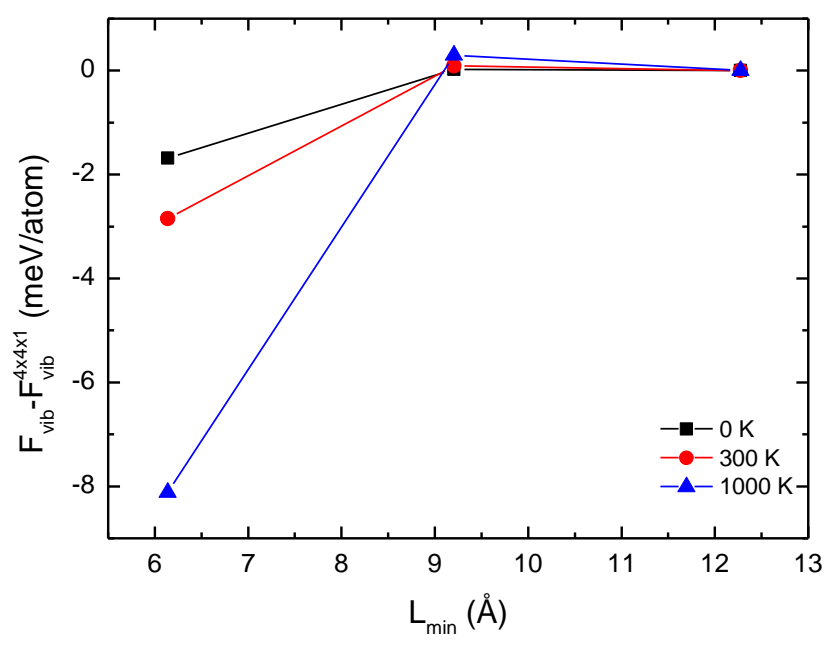

FIG. 1 (color online). Normalized vibrational energy for $2 \times 2 \times 1,3 \times 3 \times 1$ and $4 \times 4 \times 1 \mathrm{Ti}_{2} \mathrm{AIC}$ supercells as a function of $L_{\min }$, the shortest side of the supercell.

The electronic contribution $F_{e l}$ for each phase was calculated through a stepwise increase of the Fermi smearing of the electronic states (to simulate an increase in temperature), using VASP.

The QHA was implemented for selected phases by choosing a set of 6-8 volumes in steps of approximately $\pm 2 \%$ about the $0 \mathrm{~K}$ equilibrium volume, and then, at each of these volumes, performing a structural relaxation to the nearest local energy minimum. The relaxed structures were then expanded into supercells in order to calculate the force constants and hence $F_{v i b}$. Also, $F_{e l}$ was calculated at each volume. To find the global energy minimum and the corresponding volume at a given temperature, the three contributions $E_{0}, F_{e l}, F_{v i b}$ were summed at each volume, followed by a fitting procedure. 
All phases included in this work, together with their respective supercell sizes and the k-grid sizes used for both the structural relaxations of the conventional unit cells as well as for the calculations of the force constants, are listed in Table I. Except for $\mathrm{Ti}_{4} \mathrm{AlC}_{3}$, which is a hypothetical phase, all phases in the table have been experimentally verified.

TABLE I. The phases considered in this work, their respective space groups, the k-point grid for the conventional unit cell (used for volume relaxation), and the supercell sizes and supercell k-point grids for the phonon calculations.

\begin{tabular}{|c|c|c|c|c|}
\hline Phase & Space group & $\begin{array}{c}\text { k-point grid } \\
\text { conventional unit cell }\end{array}$ & $\begin{array}{c}\text { Supercell size } \\
\text { (\#atoms) }\end{array}$ & $\begin{array}{l}\text { k-point grid } \\
\text { supercell }\end{array}$ \\
\hline $\mathrm{Ti}(\mathrm{rt})$ & $\mathrm{P}_{3} / \mathrm{mmc}(\# 194)$ & $27 \times 27 \times 13$ & $3 \times 3 \times 2(36)$ & $7 \times 7 \times 7$ \\
\hline $\mathrm{Ti}(\mathrm{ht})$ & $\operatorname{Im}-3 \mathrm{~m}(\# 229)$ & $24 \times 24 \times 24$ & $4 \times 4 \times 4(64)$ & $6 \times 6 \times 6$ \\
\hline $\mathrm{Al}$ & Fm-3m (\#225) & $30 \times 30 \times 30$ & $4 \times 4 \times 4(64)$ & $6 \times 6 \times 6$ \\
\hline $\mathrm{C}$ & $\mathrm{P}_{3} / \mathrm{mmc}(\# 194)$ & $25 \times 25 \times 9$ & $4 \times 4 \times 2(128)$ & $3 \times 3 \times 3$ \\
\hline $\mathrm{Ti}_{3} \mathrm{Al}$ & $\mathrm{P}_{3} / \mathrm{mmc}(\# 194)$ & $11 \times 11 \times 15$ & $3 \times 3 \times 3(216)$ & $3 \times 3 \times 3$ \\
\hline TiAl & $\mathrm{P} 4 / \mathrm{mmm}(\# 123)$ & $23 \times 23 \times 23$ & $4 \times 4 \times 3(96)$ & $6 \times 6 \times 8$ \\
\hline $\mathrm{TiAl}_{2}(\mathrm{rt})$ & $\mathrm{I} 4_{1} /$ amd O2 (\#141) & $15 \times 15 \times 3$ & $2 \times 2 \times 1(96)$ & $7 \times 7 \times 3$ \\
\hline $\mathrm{TiAl}_{2}(\mathrm{ht})$ & Cmmm (\#65) & $23 \times 23 \times 7$ & $2 \times 1 \times 2(48)$ & $5 \times 3 \times 5$ \\
\hline $\mathrm{TiAl}_{3}$ & I4/mmm (\#139) & $25 \times 25 \times 19$ & $3 \times 3 \times 3(108)$ & $5 \times 5 \times 5$ \\
\hline $\mathrm{Ti}_{2} \mathrm{C}$ & $\mathrm{Fd}-3 \mathrm{~m}(\# 227)$ & $19 \times 19 \times 19$ & $3 \times 3 \times 3$ & $3 \times 3 \times 3$ \\
\hline $\mathrm{TiC}_{0.875}$ & Fm-3m (\#225) & $21 \times 21 \times 21$ & $2 \times 2 \times 2(120)$ & $3 \times 3 \times 3$ \\
\hline $\mathrm{TiC}$ & Fm-3m (\#225) & $23 \times 23 \times 23$ & $5 \times 5 \times 5(250)$ & $5 \times 5 \times 5$ \\
\hline $\mathrm{Al}_{4} \mathrm{C}_{3}$ & R-3m h (\#166) & $21 \times 21 \times 7$ & $3 \times 3 \times 3(189)$ & $3 \times 3 \times 3$ \\
\hline $\mathrm{Ti}_{3} \mathrm{AlC}$ & $\mathrm{Pm}-3 \mathrm{~m}(\# 221)$ & $23 \times 23 \times 23$ & $4 \times 4 \times 4(320)$ & $6 \times 6 \times 6$ \\
\hline $\mathrm{Ti}_{2} \mathrm{AlC}$ & $\mathrm{P}_{3} / \mathrm{mmc}(\# 194)$ & $23 \times 23 \times 7$ & $4 \times 4 \times 1(128)$ & $5 \times 5 \times 5$ \\
\hline $\mathrm{Ti}_{3} \mathrm{AlC}_{2}$ & $\mathrm{P}_{3} / \mathrm{mmc}(\# 194)$ & $23 \times 23 \times 5$ & $3 \times 3 \times 1(108)$ & $9 \times 9 \times 5$ \\
\hline $\mathrm{Ti}_{4} \mathrm{AlC}_{3}$ & $\mathrm{P}_{3} / \mathrm{mmc}(\# 194)$ & $17 \times 17 \times 5$ & $4 \times 4 \times 1(256)$ & $3 \times 3 \times 3$ \\
\hline
\end{tabular}

Although a comprehensive set, it should be noted that several off-stoichiometric titanium carbides have been reported to exist. However, $0 \mathrm{~K}$ calculations have shown that the most stable titanium carbide is stoichiometric TiC [33]. For this reason we have chosen $\mathrm{TiC}_{0.875}$, which is very close in 
energy to TiC, as the sole representative of an off-stoichiometric titanium carbide. We have considered both ordered and disordered vacancy configurations on the carbon sublattice for this phase. We constructed an ordered supercell comprised by $8 \mathrm{Ti}$ atoms and $7 \mathrm{C}$ atoms, and a disordered SQS cell where the corresponding numbers were 64 and 56, respectively. Nevertheless, despite the consideration of configurational entropy given by Eq. (7), neither of the $\mathrm{TiC}_{0.875}$ phases enter as a most competing phase with respect to any of the MAX phases.

\section{Results and discussion}

\section{a. $0 \mathrm{~K}$ formation enthalpies}

The $0 \mathrm{~K}$ formation enthalpies with respect to the most competing phases, $\Delta H_{c p}$, of Ti ${ }_{2} \mathrm{AlC}, \mathrm{Ti}_{3} \mathrm{AlC}_{2}$ and $\mathrm{Ti}_{4} \mathrm{AlC}_{3}$ are presented in Table II, together with their respective set of most competing phases; the results are consistent with those calculated in Ref. [5]. Both $\mathrm{Ti}_{2} \mathrm{AlC}$ and $\mathrm{Ti}_{3} \mathrm{AlC}_{2}$ have negative values of $\Delta H_{c p}$, which is usually taken as a good indicator of thermodynamic stability even though it neglects effects of temperature. $\mathrm{Ti}_{4} \mathrm{AlC}_{3}$, on the other hand, has a formation enthalpy which is very close to zero and thus smaller than the accuracy of the calculations.

TABLE III. $0 \mathrm{~K}$ formation enthalpies of $\mathrm{Ti}_{2} \mathrm{AIC}, \mathrm{Ti}_{3} \mathrm{AIC}_{2}$ and $\mathrm{Ti}_{4} \mathrm{AIC}$.

\begin{tabular}{ccc}
\hline \hline \multicolumn{3}{c}{$\mathrm{Ti}_{n+1} \mathrm{AlC}_{n}$} \\
\hline$n$ & Most competing phases & $\Delta \mathrm{H}_{\mathrm{cp}}$ (meV/atom) \\
\hline 1 & $\mathrm{Ti}_{3} \mathrm{AlC}_{2}, \mathrm{TiAl}$ & -26.7 \\
2 & $\mathrm{Ti}_{2} \mathrm{AlC}, \mathrm{Ti}_{4} \mathrm{AlC}$ & -12.3 \\
3 & $\mathrm{Ti}_{3} \mathrm{AlC}_{2}, \mathrm{TiC}$ & 0.0 \\
\hline \hline
\end{tabular}

In the following two sections we add both vibrational and electronic contributions in order to investigate whether - and if so, to which degree - the formation energies are affected as the temperature is increased above $0 \mathrm{~K}$. Since our free energy calculations of all the phases in Table I show that the sets of most competing phases over the chosen temperature interval remain those listed in Table II, the discussion below will only explicitly cover TiAl, TiC, and the three MAX phases.

\section{b. Gibbs free energies}

Figure 2 (a) shows the Gibbs free energy as a function of temperature for the 211, 312 and 413 MAX phases, and for the two binary phases TiAl and TiC. As is clearly seen in the figure, all curves follow similar trends as the temperature is increased, with $G$ smoothly decreasing at a rate which varies only slightly between the different phases. At $1000 \mathrm{~K}$, the greatest drop in $G$, in both absolute and relative terms, occurs for TiAl at about $0.353 \mathrm{eV} /$ atom (5.8\%), while the drop for TiC is the smallest at $0.221 \mathrm{eV} /$ atom ( $2.4 \%$ ), see Table III. Figure 2 (a) further shows that the difference between the $G^{\mathrm{QHA}}$ (solid lines) and $G^{\mathrm{HA}}$ (dashed lines) for each phase is relatively small; it is only at about $1000 \mathrm{~K}$ that the lines start to diverge visibly on the chosen energy scale. In panel (b) of Fig. 2 this difference is displayed as a function of temperature, and it is seen that the magnitude lies within the meV range for all five phases. At $1000 \mathrm{~K}$ the magnitude of $G^{\mathrm{QHA}-\mathrm{HA}}$ is still very nearly the same for TiC and the 
three MAX phases, while it is somewhat greater for TiAl. Beyond $1000 \mathrm{~K}$, TiC diverges from the MAX phases.
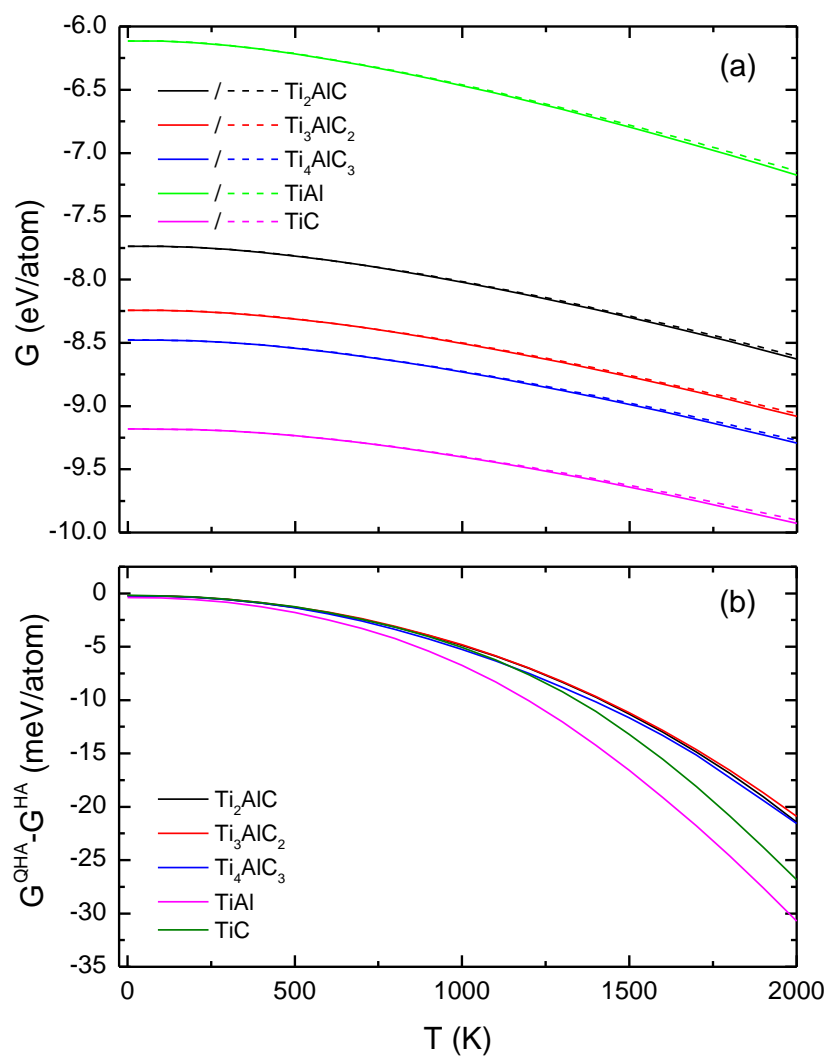

FIG. 2 (color online). (a) Gibbs free energy as a function of temperature for $\mathrm{Ti}_{2} \mathrm{AIC}, \mathrm{Ti}_{3} \mathrm{AIC}_{2}, \mathrm{Ti}_{4} \mathrm{AIC}, \mathrm{TiAl}$ and TiC. Solid lines represent QHA results, while dashed lines are the results from HA. (b) Difference in Gibbs free energy between QHA and HA.

TABLE III. Temperature dependence of the Gibbs free energy between 0 and $1000 \mathrm{~K}$ (from strongest to weakest).

\begin{tabular}{cccc}
\hline & \multicolumn{2}{c}{$G^{\mathrm{QHA}}(\mathrm{eV} /$ atom $)$} \\
\cline { 2 - 3 } Phase & $0 \mathrm{~K}$ & \multirow{2}{*}{$\Delta G^{\mathrm{QHA}}(\mathrm{eV} /$ atom $)$} \\
\hline $\mathrm{TiAl}$ & -6.114 & -6.467 & $-0.353(5.8 \%)$ \\
$\mathrm{Ti}_{2} \mathrm{AlC}$ & -7.737 & -8.021 & $-0.284(3.7 \%)$ \\
$\mathrm{Ti}_{3} \mathrm{AlC}_{2}$ & -8.243 & -8.505 & $-0.262(3.2 \%)$ \\
$\mathrm{Ti}_{4} \mathrm{AlC}_{3}$ & -8.478 & -8.730 & $-0.252(3.0 \%)$ \\
$\mathrm{TiC}$ & -9.182 & -9.403 & $-0.221(2.4 \%)$ \\
\hline \hline
\end{tabular}

The electronic and vibrational contributions to the Gibbs free energy are displayed in Fig. 3 (a) and (b), respectively. Immediately noticeable is the inverse order of the curves as compared to the Gibbs free energy curves in Fig. 2 (a); for both $F_{e l}$ and $F_{v i b}$, TiC is here the phase with the highest energy, 
followed by $\mathrm{Ti}_{2} \mathrm{AlC}, \mathrm{Ti}_{3} \mathrm{AlC}_{2}, \mathrm{Ti}_{4} \mathrm{AlC}$, and TiAl. This means that while the $0 \mathrm{~K}$ Gibbs free energy of $\mathrm{TiC}$ is lower than for the other phases, it has the weakest temperature dependence.

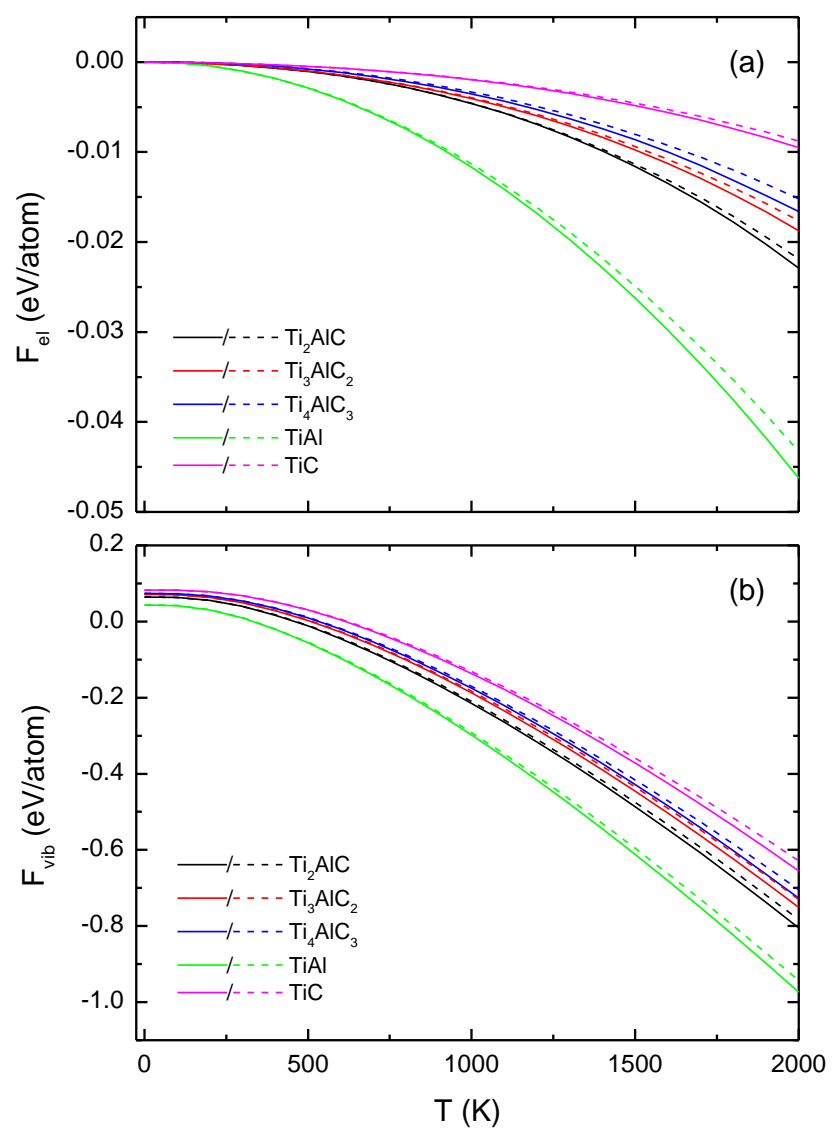

FIG. 3 (color online). (a) The electronic and (b) the vibrational contribution to the Gibbs free energy. Solid lines are the QHA results, while dashed lines are the HA results. Note the different order of magnitude on the $y$ axis between (a) and (b).

Also noticeable is the much greater relative spread in the temperature dependence of $F_{e l}$ between each phase as compared to $F_{v i b}$. However, in terms of magnitude the spread is actually greater for $F_{\text {vib, }}$ since for all five phases the temperature dependence of this contribution is much greater than for $F_{e l}$. In fact, as can be seen in Fig. 4, the temperature dependence of $G$ is almost entirely accounted for by the vibrational contribution. Neither the electronic contribution nor $E_{0}$, i.e., the $0 \mathrm{~K}$ energy (which is constant in the HA and increases slightly with temperature in the QHA), has a significant impact on the overall temperature dependence of $G$. It should be noted that Fig. 3 (a) and (b) also shows that the difference between the QHA and the HA is small, which should of course be expected from the results in Fig. 2 (a) and (b). 


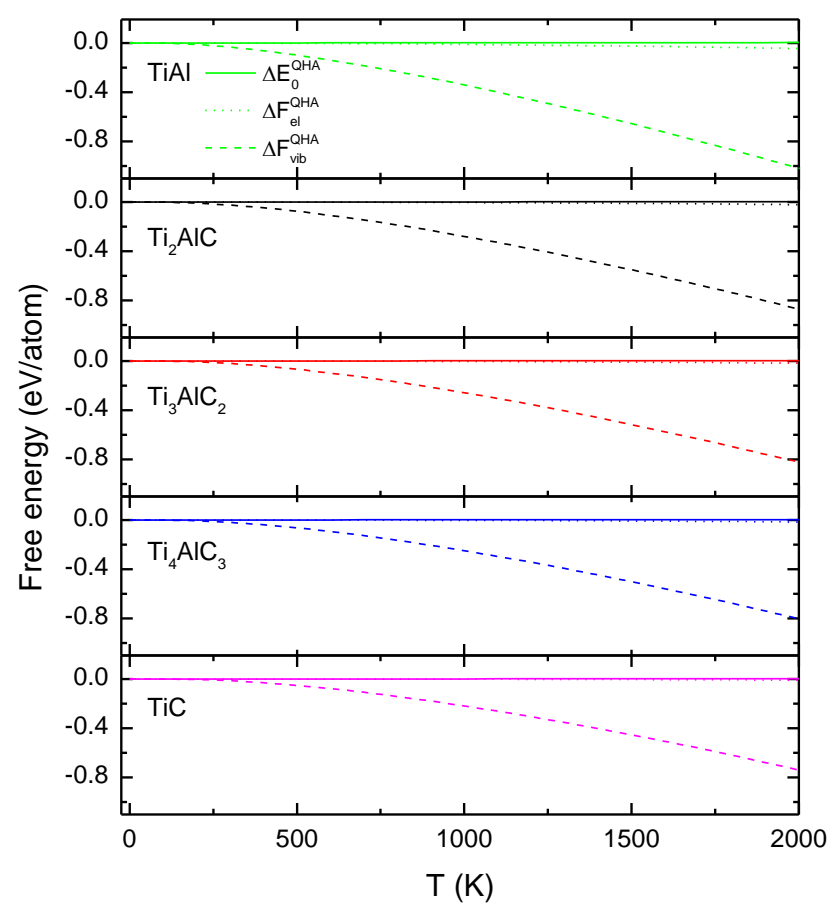

FIG. 4 (color online). A comparison between the three individual contributions to the Gibbs free energy in the QHA (normalized to the respective $0 \mathrm{~K}$ values), i.e., $E_{0}$ (solid lines), $F_{e l}$ (dotted), and $F_{v i b}$ (dashed).

The temperature dependence of both $F_{e l}$ and $F_{v i b}$, and hence $G$, strongly correlates with the Al/Ti ratio; the greater the amount of $\mathrm{Al}$ as compared to $\mathrm{Ti}$, the faster $\mathrm{G}$ decreases. This behavior can be explained by investigating the electronic and phonon density of states (EDOS and PDOS, respectively), which are presented in Fig. 5 (a) and (b).

The relevant part of the EDOS is a small region centered on the Fermi energy, since this is where the Fermi-Dirac distribution function takes on values $0<f(\varepsilon)<1$ at realistic temperatures. Thus, here the electronic entropy, which is given by Eq. (4), is greater than zero. Because the number of electronic states in this region is largest for TiAl, it has the highest entropy, and therefore the strongest temperature dependence of $F_{e l}$. The number of states is slightly lower for the three MAX phases and lowest for TiC, which explains why the temperature dependence of $F_{e l}$ becomes successively weaker with increasing C/Al ratios as seen in panel (a) of Fig. 3.

The differences in the temperature dependence of $F_{\text {vib }}$ are explained by the right term in Eq. (5): as the frequency increases, the argument of the logarithm goes to 1 , which means that lower frequencies contribute more to $F_{\text {vib }}$ than higher ones. Since the number of vibrational states at low frequencies (the acoustic modes) is largest for TiAl, followed by $\mathrm{Ti}_{2} \mathrm{AlC} \mathrm{Ti}_{3} \mathrm{AlC}_{2}, \mathrm{Ti}_{4} \mathrm{AlC}_{3}$ and TiC, the temperature dependence of $F_{v i b}$ consequently is strongest for TiAl and then becomes successively weaker for the other four phases. The reason why all phases except TiAl have vibrational states in the high frequency region (the optical modes, starting at about $15 \mathrm{THz}$ ), is that these states are dominated by vibrations of the much lighter carbon atoms [34]. Furthermore, the high frequency region helps explain the ordering in Fig. 3 (b) of the five $F_{v i b}$ curves with respect to energy: in Eq. (5) the zero point energy term is directly proportional to the sum of the phonon frequencies, thus increasing the energy of phases allowing for higher frequencies. Since the number of states in this region increases monotonously in going from TiAl to TiC, the zero point energy, and hence $F_{\text {vib, }}$ increases as well. 

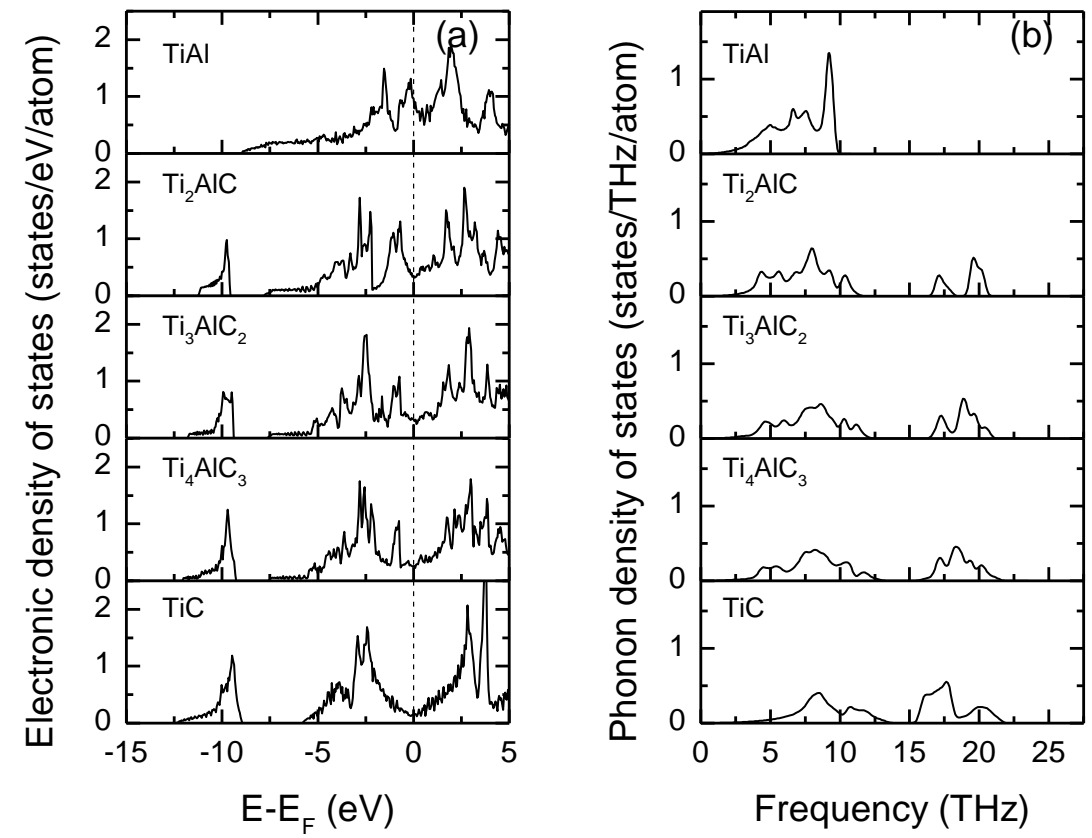

FIG. 5. (a) Electronic density of states for $\mathrm{TiAl}, \mathrm{Ti}_{2} \mathrm{AIC}, \mathrm{Ti}_{3} \mathrm{AIC}{ }_{2}, \mathrm{Ti}_{4} \mathrm{AlC}_{3}$ and $\mathrm{TiC}$. The vertical dashed line marks the Fermi energy. b) Phonon density of states.

\section{c. Phase stability}

In Fig. 6 (a) both the QHA (solid lines) and the HA (dashed lines) values of $\Delta G_{c p}$ are displayed as a function of temperature for the three $M A X$ phases. For $\mathrm{Ti}_{2} \mathrm{AlC}$ and $\mathrm{Ti}_{3} \mathrm{AlC}_{2}$ there is a slight upward trend in $\Delta G_{c p}$ which is somewhat more pronounced for the QHA than for the HA. At $1000 \mathrm{~K}, \Delta G_{c p}^{Q H A}$ for $\mathrm{Ti}_{2} \mathrm{AlC}$ has increased about $1.2 \mathrm{meV} /$ atom, while the corresponding number for $\mathrm{Ti}_{3} \mathrm{AlC}_{2}$ is 0.64 $\mathrm{meV} /$ atom. For $\mathrm{Ti}_{4} \mathrm{AlC}_{3}, \Delta G_{c p}^{Q H A}$ is even less affected, staying virtually constant throughout the entire range of temperatures. Furthermore, for this phase there is no systematic difference in behavior between $\Delta G_{c p}^{Q H A}$ and $\Delta G_{c p}^{H A}$.

The $\Delta G_{c p}$ trends within both the QHA and the HA are fully explained by the fact that, as was discussed in Sec. III (b), the temperature dependence of the Gibbs free energy is slightly different for each phase. Similarly, for a given MAX phase the differences in the trends between the two approximations can be explained by the change in temperature dependence for each phase, something which was also discussed in Sec. III (b).

Figure 6 (b)-(d) shows the relative importance of each contribution to $\Delta G_{c p}^{Q H A}$, i.e., $\Delta E_{0, c p}, \Delta F_{e l, c p}$, and $\Delta F_{v i b, c p}$, where each term is the difference between the $(0 \mathrm{~K}$, electronic, vibrational) energy of a $M A X$ phase and the corresponding set of most competing phases (cp). 


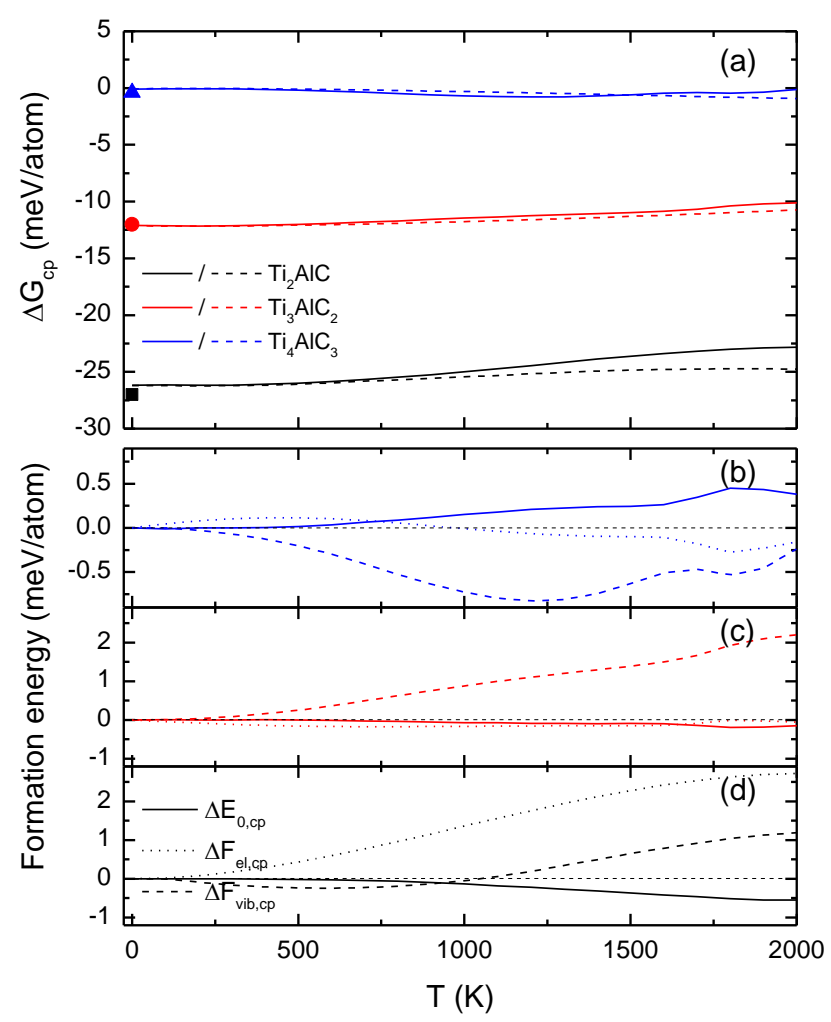

FIG. 6 (color online). (a) Difference between the Gibbs free energy for the three $\mathrm{Ti}_{n+1} A \mathrm{AC}_{\mathrm{n}} \mathrm{MAX}$ phases and their respective sets of competing phases. $\Delta G_{c p}^{Q H A}$ (solid lines) and $\Delta G_{c p}^{H A}$ (dashed lines). Symbols mark the $0 \mathrm{~K}$ values from Ref. [5]. (b)-(d) The normalized contributions to $\Delta G_{c p}^{Q H A}$, i.e., $\Delta E_{0, c p}$ (solid lines), $\Delta F_{e l, c p}$ (dotted), and $\Delta F_{v i b, c p}\left(\right.$ dashed), for $\mathrm{Ti}_{4} \mathrm{AlC}_{3}$, $\mathrm{Ti}_{3} \mathrm{AlC}_{2}$, and $\mathrm{Ti}_{2} \mathrm{AlC}$, respectively.

Panel (b) in Fig. 6 shows why $\Delta G_{c p}^{Q H A}$ for $\mathrm{Ti}_{4} \mathrm{AlC}_{3}$ is very close to being completely independent of temperature: in addition to being small, the three contributions offset each other. For $\mathrm{Ti}_{3} \mathrm{AlC}_{2}$ in Fig. 6 (c), both $\Delta E_{0, c p}$ and $\Delta F_{e l, c p}$ decrease the energy, which is compensated for by an increase in $\Delta F_{v i b, c p}$. However, since this increase is still relatively small, the observed total increase in $\Delta G_{c p}^{Q H A}$ is small as well. In Fig. 6 (d), we see that for $\mathrm{Ti}_{2} \mathrm{AlC}$, the term $\Delta E_{0, c p}$ is giving a stabilizing contribution at high temperatures, but this is more than cancelled by the increase in $\Delta F_{v i b, c p}$. The largest contribution to the temperature dependence of $\Delta G_{c p}^{Q H A}$ for $\mathrm{Ti}_{2} \mathrm{AlC}$ is coming from $\Delta F_{e l, c p}$, which increases with temperature. Thus, even though $F_{\text {vib }}$ affects $G$ to a much greater degree than $F_{e l}$ in terms of magnitude, as seen in Fig. 4 , this does not necessarily carry over to $\Delta G_{c p}$. What also matters is the temperature dependence of the $E_{0}, F_{e l}$ and $F_{v i b}$ terms for the relevant competing phases, where, due to cancellations, terms like $F_{e l}$ that are small in magnitude might nevertheless in some cases give the dominant contribution to temperature dependent changes in phase stability.

Due to the weak temperature dependence of $\Delta G_{c p}$ for the three $M A X$ phases studied here, there are no qualitative differences as compared to the $0 \mathrm{~K}$ results. As Fig. 5 (a) showed, $\Delta G_{c p}$ for both $\mathrm{Ti}_{2} \mathrm{AlC}$ and $\mathrm{Ti}_{3} \mathrm{AlC}_{2}$ stays firmly in the negative energy region even at elevated temperatures, thus correctly indicating thermodynamical stability under realistic conditions. For $\mathrm{Ti}_{4} \mathrm{AlC}_{3}$, which has so far not been found experimentally, $\Delta G_{c p}$ still remains so close to zero that a confident prediction with respect to phase stability is not warranted. Hence, while the results in this work further reinforce the conclusion in Ref. [5] that the $0 \mathrm{~K}$ energy term in the Gibbs free energy is the term mainly governing the formation of the MAX phases, we have not been able to lend any evidence to the hypothesis that the 
addition of temperature dependent contributions will have a large enough effect to resolve the energetically ambiguous case $\mathrm{Ti}_{4} \mathrm{AlC}_{3}$.

As stated above, both $\mathrm{Ti}_{2} \mathrm{AlC}$ and $\mathrm{Ti}_{3} \mathrm{AlC}_{2}$ are already known to exist, but since their respective decomposition temperatures are $1900 \mathrm{~K}$ and $\sim 1600-1700 \mathrm{~K}$ [35-37], our results should not be seen as to indicate phase stability above these temperatures; rather, they show a limitation inherent in the method used. More specifically, the method does not accommodate explicit anharmonic effects or liquids, the latter which is a disordered state and as such possessing additional configurational entropy as compared to crystal solids. If this entropic contribution is significant, it could, for example, make pure aluminum, which melts at $\sim 900 \mathrm{~K}$, or the liquid state of TiAl alloys, which melt at $\sim 1700 \mathrm{~K}$, relevant as competing phases with respect to one or more of the three MAX phases. In this hypothetical scenario, it might be that $\mathrm{Ti}_{4} \mathrm{AlC}_{3}$ is pushed well into the positive energy region. Further, since the topological disorder of liquids means that the required long-range symmetry is broken, the vibrational contribution can no longer be calculated by displacing the atoms within a supercell.

Another factor that might affect the results for $\mathrm{Ti}_{4} \mathrm{AlC}_{3}$ in particular, is crystallographic defects such as vacancies. However, based on our calculations for $\mathrm{TiC}_{0.875}$ and on calculations for off-stoichiometric $\mathrm{Ti}_{4} \mathrm{AlC}_{3}$ [38], we deem it unlikely that vacancies specifically on the carbon sublattice would dramatically affect phase stability. Furthermore, it is well known that kinetics, such as growth rate and diffusion, is important in experimental materials synthesis. However, effects thereof are beyond the scope of the present study.

A significant difference between the harmonic and quasiharmonic approximation would perhaps be expected in multicomponent systems where there are large differences between the coefficients of thermal expansion of the investigated phases and the competing phases. In such cases, it is possible that the temperature dependence of the Gibbs free energies within the QHA would deviate more strongly with respect to each other, which in turn would lead to much steeper slopes of $\Delta G_{c p}^{Q H A}$ than observed here. Conversely, in systems similar to Ti-Al-C, where the coefficients of thermal expansion vary within a relatively small interval (for $\mathrm{Ti}_{2} \mathrm{AIC}, \mathrm{Ti}_{3} \mathrm{AlC}{ }_{2}$, TiAl and $\mathrm{TiC}$ the linear coefficients of thermal expansion have been experimentally determined to $\left.7-15 \cdot 10^{-6} \mathrm{~K}^{-1}[39-42]\right), \Delta G_{c p}^{Q H A}$ for any investigated phase would be expected to remain close to $\Delta G_{c p}^{H A}$. This is a point that might be worth considering if the available computational resources are limited or if larger number of systems are to be considered, since the QHA is many times more computationally intensive than the HA.

\section{Conclusions}

We employed a first-principles based approach to study the effects of temperature on the thermodynamic phase stability of the three $M_{n+1} A X_{n}$ phases $\mathrm{Ti}_{2} \mathrm{AlC}, \mathrm{Ti}_{3} \mathrm{AlC}_{2}$, and $\mathrm{Ti}_{4} \mathrm{AlC}_{3}$ with respect to competing phases in the Ti-Al-C system. We showed that even though vibrational free energies and electronic free energies were considerable in magnitude, the formation free energies of the MAX phases with respect to competing phases were to a large degree independent of temperature. The origin is a cancellation of in particular the vibrational contribution between the MAX phases and the competing phases. This result strengthens the hypothesis put forth in earlier work that the phase stability is mainly governed by the $0 \mathrm{~K}$ energy terms. Also, we speculated that in order to resolve the energetically ambiguous case $\mathrm{Ti}_{4} \mathrm{AlC}_{3}$, various defects, explicit anharmonic effects, and liquid states may have to be taken into consideration. 


\section{Acknowledgements}

The research was funded by the European Research Council under the European Community Seventh Framework Program (FP7/2007-2013)/ERC Grant agreement no [258509]. J. Rosen acknowledges funding from the Swedish Research Council (VR) grant no. 621-2012-442, and from the Knut and Alice Wallenberg (KAW) Fellowship program. B. Alling acknowledges funding from the Swedish Research Council (VR) grant no. 621-2011-4417. The calculations were carried out using supercomputer resources provided by the Swedish National Infrastructure for Computing (SNIC) at the High Performance Computing Centre North (HPC2N).

[1] V.H. Nowotny, Progress in Solid State Chemistry, 5 (1971) 27-70.

[2] M.W. Barsoum, Progress in Solid State Chemistry, 28 (2000) 201-281.

[3] P. Eklund, M. Beckers, U. Jansson, H. Högberg, L. Hultman, Thin Solid Films, 518 (2010) 1851-1878.

[4] M. Dahlqvist, J. Rosen, S.I. Simak, D.R. McKenzie, M.M.M. Bilek, Applied Physics Letters, 97 (2010) 073103-073103.

[5] M. Dahlqvist, B. Alling, J. Rosén, Physical Review B, 81 (2010) 220102.

[6] X. Zhang, L. Yu, A. Zakutayev, A. Zunger, Advanced Functional Materials, 22 (2012) 1425-1435.

[7] Y. Wu, P. Lazic, G. Hautier, K. Persson, G. Ceder, Energy \& Environmental Science, 6 (2013) 157168.

[8] V.J. Keast, S. Harris, D.K. Smith, Physical Review B, 80 (2009) 214113.

[9] P. Eklund, M. Dahlqvist, O. Tengstrand, L. Hultman, J. Lu, N. Nedfors, U. Jansson, J. Rosén, Physical Review Letters, 109 (2012) 035502.

[10] M. Dahlqvist, B. Alling, I.A. Abrikosov, J. Rosen, Physical Review B, 84 (2011) 220403.

[11] A. Mockute, M. Dahlqvist, J. Emmerlich, L. Hultman, J.M. Schneider, P.O.Å. Persson, J. Rosen, Physical Review B, 87 (2013) 094113.

[12] A.S. Ingason, A. Petruhins, M. Dahlqvist, F. Magnus, A. Mockute, B. Alling, L. Hultman, I.A. Abrikosov, P.O.Å. Persson, J. Rosen, Materials Research Letters, 2 (2013) 89-93.

[13] A.S. Ingason, A. Mockute, M. Dahlqvist, F. Magnus, S. Olafsson, U.B. Arnalds, B. Alling, I.A. Abrikosov, B. Hjörvarsson, P.O.Å. Persson, J. Rosen, Physical Review Letters, 110 (2013) 195502.

[14] A. Togo, L. Chaput, I. Tanaka, G. Hug, Physical Review B, 81 (2010) 174301.

[15] Y. Chen, M. Chu, L. Wang, X. Bao, Y. Lin, J. Shen, physica status solidi (a), 208 (2011) 1879-1884.

[16] M.S. Ali, F. Parvin, A.K.M.A. Islam, M.A. Hossain, Computational Materials Science, 74 (2013) 119-123.

[17] M.A. Ghebouli, B. Ghebouli, A. Bouhemadou, M. Fatmi, Solid State Communications, 151 (2011) 382-387.

[18] M.A. Ghebouli, B. Ghebouli, M. Fatmi, A. Bouhemadou, Intermetallics, 19 (2011) 1936-1942.

[19] D. Music, J. Emmerlich, J.M. Schneider, Journal of Physics: Condensed Matter, 19 (2007) 136207.

[20] Z. Sun, J. Zhou, D. Music, R. Ahuja, J.M. Schneider, Scripta Materialia, 54 (2006) 105-107.

[21] J. Wang, J. Wang, Y. Zhou, Z. Lin, C. Hu, Scripta Materialia, 58 (2008) 1043-1046.

[22] G. Kresse, J. Furthmüller, Physical Review B, 54 (1996) 11169-11186.

[23] G. Kresse, J. Furthmüller, Computational Materials Science, 6 (1996) 15-50.

[24] G. Kresse, J. Hafner, Physical Review B, 47 (1993) 558-561.

[25] G. Kresse, J. Hafner, Physical Review B, 49 (1994) 14251-14269.

[26] P. Hohenberg, W. Kohn, Physical Review, 136 (1964) B864-B871.

[27] W. Kohn, L.J. Sham, Physical Review, 140 (1965) A1133-A1138.

[28] P.E. Blöchl, Phys. Rev. B, 50 (1994) 17953.

[29] J.P. Perdew, K. Burke, M. Ernzerhof, Phys. Rev. Lett., 77 (1996) 3865.

[30] J.P. Perdew, K. Burke, M. Ernzerhof, Phys. Rev. Lett., 78 (1997) 1396.

[31] X. Gonze, C. Lee, Physical Review B, 55 (1997) 10355-10368.

[32] A. Togo, F. Oba, I. Tanaka, Physical Review B, 78 (2008) 134106. 
[33] H.W. Hugosson, P. Korzhavyi, U. Jansson, B. Johansson, O. Eriksson, Physical Review B, 63 (2001) 165116.

[34] H. Li, L. Zhang, Q. Zeng, H. Ren, K. Guan, Q. Liu, L. Cheng, Solid State Communications, 151 (2011) 61-66.

[35] M.A. Pietzka, J.C. Schuster, JPE, 15 (1994) 392-400.

[36] W. Zhou, B. Mei, J. Zhu, X. Hong, J Mater Sci, 40 (2005) 2099-2100.

[37] W.K. Pang, I.M. Low, Journal of the Australian Ceramic Society 45 (2009) 30-33.

[38] M. Dahlqvist, in.

[39] J. Frodelius, E.M. Johansson, J.M. Córdoba, M. Odén, P. Eklund, L. Hultman, International Journal of Applied Ceramic Technology, 8 (2011) 74-84.

[40] N.V. Tzenov, M.W. Barsoum, Journal of the American Ceramic Society, 83 (2000) 825-832.

[41] Y. He, R.B. Schwarz, T. Darling, M. Hundley, S.H. Whang, Z.M. Wang, Materials Science and Engineering: A, 239-240 (1997) 157-163.

[42] K. Aigner, W. Lengauer, D. Rafaja, P. Ettmayer, Journal of Alloys and Compounds, 215 (1994) 121-126. 